\title{
Persistent dry cough: an unusual presentation of renal cell carcinoma
}

\author{
Stephanie Tatzel MD, Alp Sener MD PhD
}

Competing interests: None declared.

This article has been peer reviewed.

Affiliations: The Division of Urology, Department of Surgery (Tatzel, Sener) and the Department of Microbiology and Immunology (Sener), Western University, Schulich School of Medicine and Dentistry, London, Ont.

Correspondence to: Alp Sener,

alp.Sener@lhsc.on.ca

Acknowledgement: The authors thank Dr. Kathryn Roth at Western University for feedback on earlier versions of the article.

CMAJ 2014. DOI:10.1503 /cmaj.130355

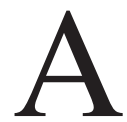
68-year-old woman presented with a 3month history of persistent, gradually worsening dry cough, which, on occasion, woke her from sleep. She had no history of hemoptysis, recent respiratory tract infection, postnasal drip, asthma, seasonal allergies, travel or constitutional symptoms. She had quit smoking 10 years earlier. Her medical history included gastroesophageal reflux disease and benign adenomas of the colon. Her current medications included rabeprazole, atorvastatin and trazodone. The results of extensive investigations, including chest radiography, spirometry, flexible nasoendoscopy and a battery of blood tests, were negative, except for microcytic anemia, which we attributed to iron deficiency (hemoglobin 107 [normal 123-157] g/L).

Six months following the initial presentation, our patient returned with worsening cough and complaints of drenching night sweats and unexplained weight loss of $5 \mathrm{lbs}$. A contrastenhanced computed tomography (CT) scan of the thorax showed prominent varicosities on the right side of the retroperitoneum. A subsequent CT of her abdomen and pelvis showed a hypervascular mass in the upper pole of the right kidney (Figure 1), with no evidence of retroperitoneal lymphadenopathy.

Our patient underwent open right radical nephrectomy for renal cell carcinoma (Führman grade 3, clear cell, with negative margins); there was no evidence of hepatic invasion. Following the surgery, the patient's cough completely resolved and did not recur during the next 2 years of follow-up.

This patient's case represents a rare cause of chronic cough and an unusual presentation of renal cell carcinoma. Renal cell carcinomas secrete prostaglandins (mainly prostaglandin $\mathrm{E}_{2}$ ), which have been implicated in enhancing the cough reflex via the $\mathrm{EP}_{3}$ receptor pathway. ${ }^{1,2} \mathrm{In}$ our patient's case, a mass effect by the tumour on the posterior diaphragm may have also contributed to irritation and reflex cough. ${ }^{3}$

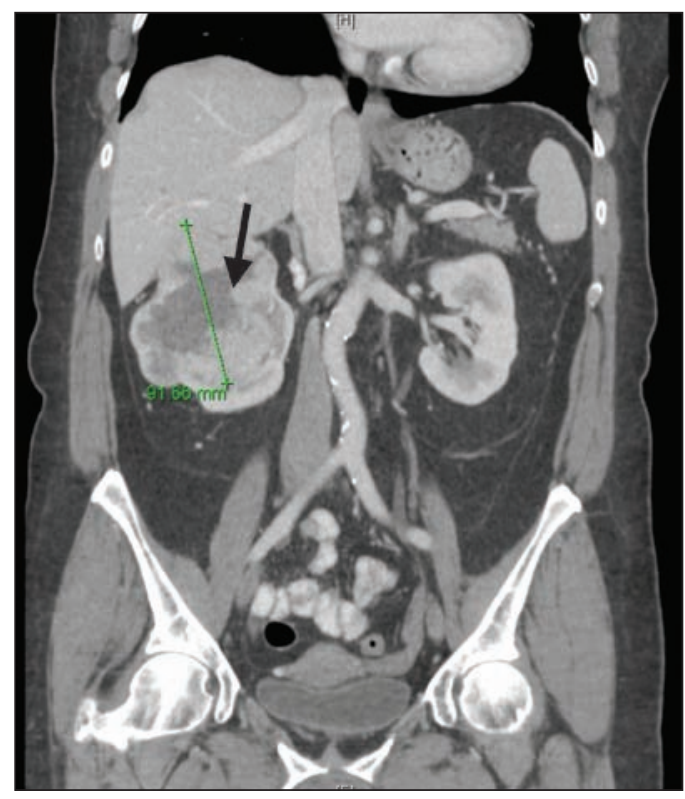

Figure 1: Computed tomograph showing a large hypervascular right renal mass associated with the upper pole (arrow) in a 68-year-old woman with cough. There are multiple tumour lobulations that indent the hepatic capsule.

\section{References}

1. Ishiura Y, Fujimura M, Nobata K, et al. Prostaglandin $I_{2}$ enhances cough reflex sensitivity to capsaicin in the asthmatic airway. Cough 2007;3:2.

2. Maher SA, Birrell MA, Belvisi MG. Prostaglandin $\mathrm{E}_{2}$ mediates cough via the $\mathrm{EP}_{3}$ receptor. Am J Respir Crit Care Med 2009; 180:923-8.

3. Chung KF, Pavord ID. Prevalance, pathogenesis and causes of chronic cough. Management of chronic cough. Lancet 2008; 371:1364-74

Clinical images are chosen because they are particularly intriguing, classic or dramatic. Submissions of clear, appropriately labelled high-resolution images must be accompanied by a figure caption and the patient's written consent for publication. A brief explanation (250 words maximum) of the educational significance of the images with minimal references is required. 